Vol. 39 (1989) [329-333]

\title{
PROPERTIES OF AXIAL DIAMETERS
}

\author{
PAUL R. ScotT
}

\begin{abstract}
$\Lambda$ is a lattice and $K$ a bounded, open, convex set in $E^{n}$. An axial diameter of $K$ is the maximal length $X_{i}$ of chords of $K$ parallel to the $i$ th lattice basis vector $(1 \leqslant i \leqslant n)$. A number of properties of the axial diameters are developed. For sets $K$ containing just one lattice point, an inequality is established; when $\Lambda$ is the integral lattice, this inequality takes the form $\sum_{i=1}^{n} X_{i}^{-1} \geqslant n /(n+1)$.
\end{abstract}

\section{INTRODUCTION}

Let $\Lambda$ be a lattice in $n$-dimensional Euclidean space $E^{n}$, having determinant $d(\Lambda)$, and being generated by the vectors $x_{1}, x_{2}, \ldots, x_{n}$. Suppose that $\left|x_{i}\right|=\xi_{i}(1 \leqslant i \leqslant n)$. Let $K$ be a bounded, open, convex body in $E^{n}$.

In the literature, there are many known inequalities relating various attributes of $K$, such as the volume, surface area, diameter and width. Some similar results have been established for bodies $K$ with lattice point constraints; see for example $[1,4,5]$. However, these results are often difficult to establish, and are in a sense unnatural, in that the measurements being compared are not related to the lattice in any particular way. Thus for lattice related inequalities, it may be more profitable to investigate inequalities involving projections on the coordinate hyperplanes, or the axial diameters defined in [6]. For each $i$, amongst all lines parallel to $x_{i}$, there exists (at least) one which intercepts $K$ in a segment of maximal length. We call this maximal length an axial diameter of $K$, and denote it by $X_{i}(K)=X_{i}(1 \leqslant i \leqslant n)$. In [6] we obtained the pretty result:

THEOREM 1. If $K$ contains no points of $\Lambda$, then

$$
\sum_{i=1}^{n} \xi_{i} X_{i}^{-1} \geqslant 1
$$

and this inequality is best possible.

One advantage of working with the axial diameters depends on the fact that ratios of lengths in the same direction are left invariant under affine transformation. This

Received 29th June 1988

Copyright Clearance Centre, Inc. Serial-fee code: 0004-9729/89 \$A2.00+0.00. 
means that a result like the above theorem is essentially independent of the particular lattice used, and there is no loss in generality in taking $x_{i}$ to be the unit vector $e_{i}$ for each $i,(1 \leqslant i \leqslant n)$. In this case $\Lambda$ becomes the integral lattice, and $d(\Lambda)=1$. We assume henceforth that we are working with the integral lattice.

Our purpose in this paper is to establish a number of interesting properties of the axial diameters (Sections 2-4), and to establish a generalisation of Theorem 1 for convex bodies containing one lattice point (Section 5 ).

\section{CENTRE OF SYMMETRY AND CENTRE OF GRAVITY}

In this section, let $K$ be a bounded, open, convex body in $E^{n}$, having centre of gravity $G$. The following result is stated by Ehrhart [2], and an outline proof for convex bodies in $E^{n}$ is given by Hammer in [3].

LEMMA 1. In $E^{n}$, any chord of $K$ which passes through the centre of gravity, $G$, of $K$, is partitioned by $G$ into two segments; the ratio of the lengths of these segments lies between $1 / n$ and $n$, and each of these bounds can be attained.

TheOREM 2. Suppose that $K$ contains the origin $O$, but no non-zero points of the integral lattice.

(a) If $K$ is symmetric about the origin, then $X_{i} \leqslant 2(1 \leqslant i \leqslant n)$.

(b) If $K$ has its centre of gravity at the origin, then $X_{i} \leqslant n+1(1 \leqslant i \leqslant n)$.

Proof: The proof of $(\mathrm{a})$ is trivial. For given $i, K$ does not contain the lattice points $\pm \mathbf{e}_{i}$; since $K$ is convex, $K$ is bounded by hyperplanes through these two points. Further, since $K$ is symmetric about $O$, we may assume that these supporting hyperplanes are parallel. Since the distance between them is at most 2 , it follows that $X_{i} \leqslant 2$.

In case (b), for given $i, K$ does not contain the point $-\mathbf{e}_{i}$. Hence, as above, $K$ is bounded by a hyperplane $H$ through this point. We assert that $K$ is also bounded by the parallel hyperplane $H^{\prime}$ through the point $n \mathbf{e}_{i}$. For suppose this is not true. Then $K$ has a boundary point $A$ such that $A$ and $O$ are separated by $H^{\prime}$. Let $A O$ meet the boundary of $K$ again in $B$, and the hyperplanes $H, H^{\prime}$ in $Q, Q^{\prime}$ respectively. By Lemma $1 O B / O A \geqslant 1 / n$. But by the definition of the hyperplanes, $O B / O A<O Q / O Q^{\prime}=1 / n$. This contradiction establishes our result.

COROLLARY. If $K$ has centre of gravity at the origin, then

$$
\sum_{i=1}^{n} \frac{1}{X_{i}} \geqslant \frac{n}{n+1}
$$

\section{AXIAL DIAMETERS AND SUPPORTING HYPERPLANES}

It is interesting to ask whether the axial diameters share any of the properties of the more commonly defined diameters. It is well-known that the supporting hyperplanes 
to $K$ at the endpoints of a diameter are always parallel. The traditional proof allows the possibility of the existence of a diameter in any direction. The result remains true for any axial diameter, but we require a different proof.

TheOREM 3. The supporting hyperplanes to $K$ at the endpoints of any axial diameter are parallel.

Proof: Let $A B$ be an axial diameter of $K$ in the $x_{i}$-direction, having length $X_{i}$. Then since $K$ is open, for all points $\mathbf{u}, \mathbf{v} \in K$ with $u_{j}=v_{j}(1 \leqslant j \leqslant n, j \neq i)$, we have $\left|u_{i}-v_{i}\right|<X_{i}$.

Now translate $K$ through $\overrightarrow{A B}$ to position $K_{T}$. We assert that $K \cap K_{T}=\emptyset$. For if not, there exists a vector $\mathbf{v} \in K \cap K_{T}$ which arises from a $\mathbf{u} \in K$. For this $\mathbf{u}, \mathbf{v}, u_{j}=v_{j}(1 \leqslant j \leqslant n, j \neq i)$, and $u_{i}+X_{i}=v_{i}$. But now $\mathbf{u}, \mathbf{v} \in K$, and $\left|u_{i}-v_{i}\right|=$ $X_{i}$, a contradiction.

Hence, since $K, K_{T}$ are open and convex, and $K \cap K_{T}=\emptyset$, there exists a hyperplane which separates $K$ and $K_{T}$. By construction, this hyperplane passes through the point $B$ on the boundary of each. We deduce that $K$ is supported by parallel hyperplanes at the endpoints $A$ and $B$ of the axial diameter.

ConollakY. The supporting hyperplanes to $K$ at the endpoints of a diameter are parallel.

Proof: This follows immediately by regarding the diameter as the axial diameter in that direction.

\section{Axial Diameters and VOLUME}

We can use the axial diameters to provide a lower bound for the volume of $K$.

ThEOREM 4. If $K$ is a convex body in $E^{n}$ with volume $V$ and axial diameters $X_{1}, X_{2}, \ldots, X_{n}$, then

$$
\frac{1}{n !} X_{1} X_{2} \ldots X_{n} \leqslant V
$$

Proof: We symmetrise $K$ about each of the coordinate hyperplanes in turn to obtain the symmetral $K^{*}$. By the properties of Steiner symmetrisation,

$$
V\left(K^{*}\right)=V(K), X_{i}\left(K^{*}\right) \geqslant X_{i}(K)(1 \leqslant i \leqslant n) .
$$

Now for $K^{*}$, an axial diameter in each direction lies along the corresponding axis, and is bisected by the origin. Since $K^{*}$ is convex, $K^{*}$ contains the convex hull of these $n$ axial diameters, a generalised octahedron of volume $X_{1}^{*} X_{2}^{*} \ldots X_{n}^{*} / n$ !. Hence

$$
V(K)=V\left(K^{*}\right) \geqslant \frac{1}{n !} X_{1}^{*} X_{2}^{*} \ldots X_{n}^{*} \geqslant \frac{1}{n !} X_{1} X_{2} \ldots X_{n}
$$


as required.

We observe that no similar upper bound exists for the volume. For example, in $E^{2}$, the infinite strip bounded by $y=x, y=x+1$ has infinite area ("volume"), and unit axial diameters.

\section{Convex Bodies Containing one lattice point}

We now establish a generalisation of Theorem 1 .

THEOREM 5. If $K$ is a convex set in $E^{n}$ which contains the origin, but no non-zero points of the integral lattice, then

$$
\sum_{i=1}^{n} \frac{1}{X_{i}} \geqslant \frac{n}{n+1}
$$

This result is best possible: it is attained for the simplex $\Delta_{n}$ having vertices at the origin and the $n$ lattice points $(n+1) \mathbf{e}_{i}(1 \leqslant i \leqslant n)$.

Proof: The proof is similar to the proof of Theorem 1 given in [6]. We symmetrise the set $K$ about each of the coordinate hyperplanes in turn, to obtain a new set $K^{*}$. Such symmetrisation does not decrease the axial diameters. Also, since $K$ contains no lattice points on the hyperplanes $x_{i}= \pm 1$, so $K^{*}$ contains none of the points $\left( \pm 1, \pm \frac{1}{2}, \ldots, \pm \frac{1}{2}\right)^{\prime}$, where the prime indicates all possible pernutations of the coordinates. In particular, in the positive orthant, $K^{*}$ contains none of the points $\left(1, \frac{1}{2}, \ldots, \frac{1}{2}\right)^{\prime}$. Hence by convexity it does not contain the centroid $\frac{1}{n} \sum\left(1, \frac{1}{2}, \ldots, \frac{1}{2}\right)^{\prime}=((n+1) / 2 n, \ldots(n+1) / 2 n)$ of the $(n-1)$-dimensional determined simplex. Using $X_{i}(1 \leqslant i \leqslant n)$ to denote the axial diameters of $K^{*}, K^{*}$ contains the $n$ points $\left(\frac{1}{2} X_{1}, 0, \ldots, 0\right), \ldots,\left(0,0, \ldots, \frac{1}{2} X_{n}\right)$. The condition that the hyperplane determined by these $n$ points separates the above centroid from the origin easily simplifies to

$$
\left|\begin{array}{ccccc}
X_{1} & 0 & \ldots & 0 & 1 \\
0 & X_{2} & \ldots & 0 & 1 \\
\vdots & \vdots & \ddots & \vdots & \vdots \\
0 & 0 & \ldots & X_{n} & 1 \\
1 & 1 & \ldots & 1 & \frac{n}{n+1}
\end{array}\right| \leqslant 0
$$

Replacing row $(n+1)$ by row $(n+1)-\sum_{i=1}^{n}\left(X_{i}^{-1} \times\right.$ row $\left.i\right)$, we obtain immediately the required condition.

Notice that Corollary 1 of Theorem 2 gives a special instance of this result. 


\section{REFERENCES}

[1] J.R. Arkinstall and P.R. Scott, 'An isoperimetric problem with lattice point constraints', $J$. Austral. Math. Soc. (A) 27 (1979), 27-36.

[2] E. Ehrhart, 'Sur les ovales et les ovöides', C.R. Acad. Sci. Paris 240 (1955), 583-585.

[3] P.C. Hammer, 'The centroid of a convex body', Proc. Amer. Math. Soc. 2 (1951), 522-525.

[4] P. McMullen and J.M. Wills, 'Minimal width and diameter of lattice point free convex bodies', Mathematika 28 (1981), 255-264.

[5] P.R. Scott, 'Two inequalities for convex sets with lattice point constraints in the plane', Bull. London Math. Soc. 11 (1979), 273-278.

[6] P.R. Scott, 'Lattices and convex sets in space', Quart. J. Math. 36 (1985), 359-362.

[7] P.R. Scott, 'On the volume and projection of convex sets containing no lattice points', Bull. Austra. Math. Soc. 32/3 (1985), 331-338.

Department of Mathematics

University of Adelaide

G.P.O. Box 498

Adelaide 5001

South Australia

Australia. 